九州大学学術情報リポジトリ

Kyushu University Institutional Repository

\title{
Nutrient limitation alters the levels of two distinct forms of cyclin-dependent kinase G2, a putative autophagy progression modulator in tobacco BY-2 cells
}

\section{NAGAHAMA, Masaki}

Laboratory of Plant Nutrition, Division of Soil Science and Plant Nutrition, Department of Plant Resources, Graduate School of Bioenvironmental Sciences, Kyushu University

ASATSUMA, Satoru

Laboratory of Plant Nutrition, Division of Soil Science and Plant Nutrition, Department of Plant Resources, Faculty of Agriculture, Kyushu University

SHIMIZU, Masami

Laboratory of Horticultural Science, Division of Agrobiological, Sciences, Department of Bioresource Sciences, Faculty of Agriculture, Kyushu University

MATSUOKA, Ken

Laboratory of Plant Nutrition, Division of Soil Science and Plant Nutrition, Department of Plant Resources, Graduate School of Bioenvironmental Sciences, Kyushu University

https://doi.org/10.5109/4363545

出版情報: 九州大学大学院農学研究院紀要. 66 (1)，pp.1-10，2021-03-01. Faculty of Agriculture， Kyushu University

バージョン :

権利関係 : 


\title{
Nutrient limitation alters the levels of two distinct forms of cyclin-dependent kinase G2, a putative autophagy progression modulator in tobacco BY-2 cells
}

\author{
Masaki NAGAHAMA ${ }^{1}$, Satoru ASATSUMA ${ }^{2,3}$, Masami SHIMIZU $^{3}$ \\ and Ken MATSUOKA ${ }^{1,2,3, *}$ \\ Laboratory of Plant Nutrition, Division of Molecular Biosciences, Department of Bioscience and Biotechnology, \\ Faculty of Agriculture, Kyushu University, Fukuoka 819-0395, Japan \\ (Received October 8, 2020 and accepted November 4, 2020)
}

\begin{abstract}
By screening protein kinases that retard autophagy progression in tobacco (Nicotiana tabacum) BY-2 cells, we found that a tobacco cyclin-dependent kinase G2 (NtCDKG2) would be involved in autophagy progression under sucrose-starvation condition. Characterization of its cDNA, and comparison with corresponding mRNA and genome sequences in parental species of tobacco indicated that the NtCDKG2 gene originated from $N$. tomentosiformis. Comparison of the deduced amino acid sequences of NtCDKG2 cDNA with those of $N$. tomentosiformis indicated that the protein encoded by the cDNA (NtCDKG2;1) is identical to a shorter variant of the CDKG2 protein. Characterization of NtCDKG2;1 protein using specific antibodies indicated that $73 k D a$ and $48 k D a$ forms, designated as NtCDKG2; $1^{\text {p73 }}$ and NtCDKG2; $1^{148}$, present in tobacco BY-2 cells. NtCDKG2; $1^{\mathrm{p} 73}$ decreased under starvation of either sucrose or phosphate whereas NtCDKG2;1 $1^{\mathrm{p} 48}$ decreased under starvation of sucrose and nitrogen. These data suggest that the roles of these two forms differ in tobacco cells.
\end{abstract}

Key words: alternative splicing, cell cycle, starvation, sucrose

\section{INTRODUCTION}

Plants have various ways of responding to limitations in the supply of nutrients and energy. One well known response is the suppression of growth. Another response that has been studied in recent years is the induction of autophagy, which is the digestion by a cell of its own intracellular contents (Avila-Ospina et al., 2014). It has been shown that this reaction occurs at the cellular level, since autophagy can be induced in cultured cells by replacing the regular medium with a nutrient-deficient medium. In the case of tobacco BY-2 cells, autophagy can be induced under limitations of sugar, nitrogen $(\mathrm{N})$, or phosphate $(\mathrm{Pi})$ in the medium (Moriyasu and Ohsumi, 1996; Toyooka et al., 2006; Toyooka and Matsuoka, 2006; Matsuoka 2009; Tasaki et al., 2014).

It has been shown that Pi starvation responses that take several days are linked to sugar-signaling pathways in intact plants (Rouached et al., 2010). In contrast, less than $12 \mathrm{~h}$ are sufficient for the induction of autophagy in tobacco BY-2 cells under sugar, nitrogen or phosphatedeficient conditions (Moriyasu and Ohsumi, 1996; Toyooka et al., 2006; Tasaki et al., 2014). It has also been shown in tobacco BY-2 cells that not only autophagy but also the suppression of the progress in cell cycle is induced by Pi starvation (Sano et al., 2004).

In contrast to Pi starvation, the starvation of other

\footnotetext{
Laboratory of Plant Nutrition, Division of Soil Science and Plant Nutrition, Department of Plant Resources, Graduate School of Bioenvironmental Sciences, Kyushu University

${ }^{2}$ Laboratory of Plant Nutrition, Division of Soil Science and Plant Nutrition, Department of Plant Resources, Faculty of Agriculture, Kyushu University

${ }^{3}$ Cell Function Research Team, RIKEN Plant Science Center

* corresponding author (E-mail: kenmat@agr.kyushu-u.ac.jp)
}

major nutrients does not stop the cell cycle of tobacco cells at a specific phase, although it does arrest cell growth as in the case of Pi starvation (Sano et al., 2004). We have recently shown that the pathway for autophagy induction upon Pi starvation is different from that of $\mathrm{N}$ and sugar starvation (Tasaki et al., 2014).

It is well documented in yeast and mammals that cell cycle regulation and nutrient-dependent autophagyinduction are mediated by common regulatory proteins. For example, a yeast protein kinase called target of rapamycin, as well as its mammalian orthologs are involved in both cell cycle and autophagy regulations (Kamada et al., 2004; Mathiassen et al., 2017). The plant ortholog of target of rapamycin also regulates cell growth, sugar and energy metabolism (Xiong and Sheen, 2014) and autophagy (Liu and Bassham, 2010). Yet, we still do not have enough knowledge about players that regulate the autophagy progression under starvation of different nutrients in plants.

We showed previously that a protein aggregate formed from the fusion protein of cytochrome b5 (Cyt b5) and red fluorescence protein (RFP), which is designated as Cyt b5-RFP, can be a model substrate allowing monitoring of autophagy in tobacco BY-2 cells (Toyooka et al., 2006; Matsuoka 2009; Klionsky et al., 2008). We also showed that progress of autophagy can be quantitatively scored by tracing the fates of intact and processed forms of Cyt b5-RFP, which are separated by SDSPAGE, since this processing is reportedly resulted after the induced onset of autophagy (Toyooka et al., 2006).

To gain insights into the induction of autophagy in relation to the cell cycle, we screened protein kinases related to the cell cycle or metabolic regulation, that have been found in the tobacco EST collection (Matsuoka et al., 2004; Gális et al., 2006), through the antisense method combined with the Cyt b5-RFP aggre- 
gate processing assay. Here, we report that tobacco cyclin dependent protein kinase G2 (NtCDKG2), which is a member of the cyclin-dependent kinase family with significant similarity to mammalian PITSLRE/CDK11 kinase, seems to be involved in autophagy progression under sucrose-starvation condition. We also report that this kinase has two forms in tobacco cells, and the levels of the two forms are differentially regulated under distinct major nutrient starvation conditions.

\section{MATERIALS AND METHODS}

The wild-type line of tobacco BY-2 cells and transformed BY-2 cells expressing Cyt b5-RFP fusion protein were cultured in normal medium or in nutrient-deficient media as described (Toyooka et al., 2006). For antisense analysis, cDNA fragments for protein kinases were selected from the EST collection of tobacco BY-2 cells (Matsuoka et al., 2004; Gális et al., 2006) and placed in the antisense direction under the control of an enhancerduplicated CaMV35S promoter in an expression vector pMAT137-Hm. The pMAT137-Hm vector is a derivative of the expression vector pMAT137 (Yuasa et al., 2005) containing a CaMV35S promoter-hygromycin phosphotransferase-nos terminator cassette from pIG121-Hm (Ohta et al., 1990) inserted into the Cla I restriction site of the pMAT137. The resulting plasmids were introduced into Agrobacterium tumefaciens EHA101 (Hood et al., 1986) by electroporation and used for the transformation of BY-2 cells expressing Cyt b5-RFP fusion protein. For the screening of transformed tobacco BY-2 cells expressing both Cyt b5-RFP and the antisense construct, $100 \mathrm{mg} / \mathrm{l}$ of hygromycin in solid medium was used. Transformed colonies that appeared on the selection plates were pooled, re-introduced into suspension culture, and subcultured weekly for three to five weeks to confirm the constant rate of growth in each transformed cell line. Thereafter, three-day-old cells after subculture, which contained high levels of dividing cells, were harvested and re-suspended into medium with or without sucrose, $\mathrm{N}$, or $\mathrm{Pi}$, and then further cultured for 24 or $48 \mathrm{~h}$. These cells were then harvest and the proteins were extracted and separated by SDS-PAGE without heat denaturing. Fluorescence images were collected from the SDS-polyacrylamide gels and the ratio of the intensity of the intact band to the sum of the intensities of intact and processed bands was calculated after quantifying the intensity of the intact and processed forms of Cyt b5-RFP. Alternatively, individual colonies were introduced into suspension as above and used for the confirmation of autophagy suppression.

To analyze the length of the tobacco cells as well as to measure the mitotic index, suspension-cultured cells in an appropriate medium were observed under an Olympus IX40 inverted light microscope and images of the cells were collected using a DP-7 camera. Thereafter, the frequency of M-phase cells among 100 cells was manually counted. The length of the tobacco BY-2 cells, which take roughly cylinder shape, was measured using ImageJ software (https://imagej.nih.gov/ij/) by quantifying the height of the cylinder.

Cloning of the full-length cDNA clone for NtCDKG2 was performed as follows. The primary full-length cDNA library obtained by using a pGCAPsp2 vector and mRNA from tobacco BY-2 cells (Kato et al., 2005; Toyooka et al., 2009), was introduced into $E$. coli and the bacterial mixture was spread on selection plates. Approximately 1,000 clones were pooled, and mixture of plasmids were prepared from the pools. Ninety-six of pooled plasmids were prepared from libraries made from mRNA prepared from the log-phase cells, the stationary phase cells, and the mixture of cells treated with either ABA or methyl jasmonate, because we worked the response of the tobacco cells against these phytohormones (Matsuoka et al., 2004; Gális et al., 2006). The pooled plasmids were placed onto 96-well deep-well plates. In total, three sets of the 96-well plates with the full-length library were generated. Then mixtures of the well plate columns and rows were prepared. Using the pooled plasmids that correspond to the mixtures of plasmids on the columns and rows as templates, 60 PCR reactions were carried out using the primers GGATTTCCCTTGACATCTCTA and CACCCAAAGCTGGAAGCTGGT. The primary pools of cDNA in the 96-well plate that gave positive signals by the PCR were identified. Then the library in the pool was re-transformed into $E$. coli and 20 sets of the pooled mini-library consisting of approximately 100 clones were prepared and used as the template for the next round of PCR. After finding that a particular pooled mini-library gave positive signals, the plasmids in that mini-library were introduced into $E$. coli and colonies of transformants were formed on a selection plate. Then colony PCR was carried out to identify the colonies that gave positive signals by PCR.

The nucleotide sequences of the identified clones were determined by the dideoxy method using appropriate primers. Then the longest open reading frame was translated into an amino acid sequence for sequence comparison. To find DNA and protein sequences related to the clone, BLASTn, BLASTp, and tBLASTn searches were carried out against non-redundant and selected databases using the NCBI website (http://blast.ncbi.nlm. nih.gov/Blast.cgi). The sequence alignment was carried out with Clustal Omega (http://www.ebi.ac.uk/Tools/msa/ clustalo/) and the results were manually edited.

To prepare cell lysate to analyze the NtCDKG2 proteins, cells cultured in an appropriate medium were harvested by filtration and suspended into an equal volume of ice-cold lysis buffer consisting of $0.45 \mathrm{M}$ sucrose, 50 mM Tris-MES pH 7.3, 2 mM DTT, and one drop of inhibitor cocktail (Roche diagnostics KK, Tokyo, Japan) per $50 \mathrm{ml}$. Cells were homogenized using a Potter-Elvehjem homogenizer as described (Matsuoka et al., 1997). The homogenate was centrifuged at $1,000 \mathrm{~g}$ for $10 \mathrm{~min}$ and the supernatant was collected as the total lysate. Then the total lysate was centrifuged at $10,000 \mathrm{~g}$ for $10 \mathrm{~min}$ and the supernatant and pellet were used as the soluble and sedimented fractions, respectively. Proteins were quantified using Bio-Rad Protein Assay (Bio-Rad Laboratories, Hercules, California, USA) using bovine 
serum albumin as standard.

Antibody against the N-terminal domain of NtCDKG2 were prepared as follows. Peptides with the sequences SSRDRGRLRQKDVKERC and SVSQSDTESEDEHDSRC, termed peptide $\mathrm{N} 1$ and $\mathrm{N} 2$ and corresponding to the $54^{\text {th }}-69^{\text {th }}$ and $360^{\text {th }}-375^{\text {th }}$ of NtCDKG2;1 protein with an additional C-terminal cysteine residue for conjugation, respectively, were chemically synthesized, mixed and then conjugated to keyhole limpet hemocyanin, and the conjugate was used to immunize New Zealand white rabbits. Anti-NtCDKG2 N-terminal domain IgG was purified from the serum by affinity purification using the column in which these peptides were conjugated. The resulting IgG was used for immunoblotting. The anti-C-terminalpeptide antibody was generated using the peptides CRSVDEFERLNRIDEGTY and CREVPLPKSKEFMPTFPA, termed peptide $\mathrm{C} 1$ and $\mathrm{C} 2$ and corresponding to $394^{\text {th }}$ $411^{\text {th }}$ of NtCDKG2;1 protein and $692^{\text {nd }}-708^{\text {th }}$ of NtomCDKG2 isoform X1 with N-terminal cysteine add for conjugation, respectively, as described above. Immunoblotting was carried out as described (Oda et al., 2020). The intensity of the signals on the immunoblot was quantified using ImageQuant software (GE healthcare). In some cases, antigen peptide(s) were included in diluted antibody solution as indicated in the legends to the figures.

\section{RESULTS}

\section{Expression of the antisense construct of NtCDKG2 kinase slows the progression of sucrose-starvation-dependent autophagy}

Based on information from EST clones from tobacco BY-2 cells (Matsuoka et al., 2004; Gális et al., 2006), we chose seven ESTs for non-transmembrane protein kinases that showed higher expression during the stationary phase of growth (Matsuoka et al., 2005), since reaching the stationary phase of growth is induced upon nutrient starvation. These antisense constructs were placed under an enhancer-duplicated CaMV35S promoter and the resulting constructs were introduced into transformed tobacco BY-2 cells expressing Cyt b5-RFP (Toyooka et al., 2006). Most of the transformation with the antisense constructs yielded over 200 colonies on the selection plates. An exception was BY11635, which encodes a part of a tobacco ortholog of yeast target of rapamycin. In this case, only a few transformed colonies appeared on the selection plates.

Exponentially growing cells in suspension culture derived from pooled transformants expressing both Cytb5-RFP and one of the antisense constructs were harvested and re-suspended into medium with or without sucrose, N, or Pi, and then further cultured for $24 h$. Proteins extracted from these cells were separated by SDS-polyacrylamide gel electrophoresis (SDS-PAGE) without heat denaturing. Fluorescence images were collected from the SDS-polyacrylamide gels and intensities of the band for intact form of Cyt b5-RFP (Fig. 1a, open arrowhead) and processed form of Cyt b5-RFP generated by autophagy (Fig. 1a, closed arrowhead) were quantified. Then the ratio of the intensity of the intact band to the sum of the intensities of intact and processed bands was calculated. The higher ratio in antisense construct-introduced cells than that in vectorintroduced cells suggests a possibility that the protein kinase corresponding to the antisense construct may be involved in autophagy.

Among the seven protein kinases tested, suppression of BY4753 retarded the formation of the processed form of Cyt b5-RFP under both sucrose and Pi starvation conditions. Nearly $40 \%$ of Cyt b5-RFP were intact size in the BY4753 suppressed cells whereas in vector control cells the level of intact size was about $20 \%$. This observation suggests that the protein kinase that is encoded by BY4753 could be involved in the progression of sugar- and Pi-depletion-dependent autophagy. Expression of suppression constructs for other protein kinases either slightly increased or did not alter the level of intact Cyt b5-RFP.
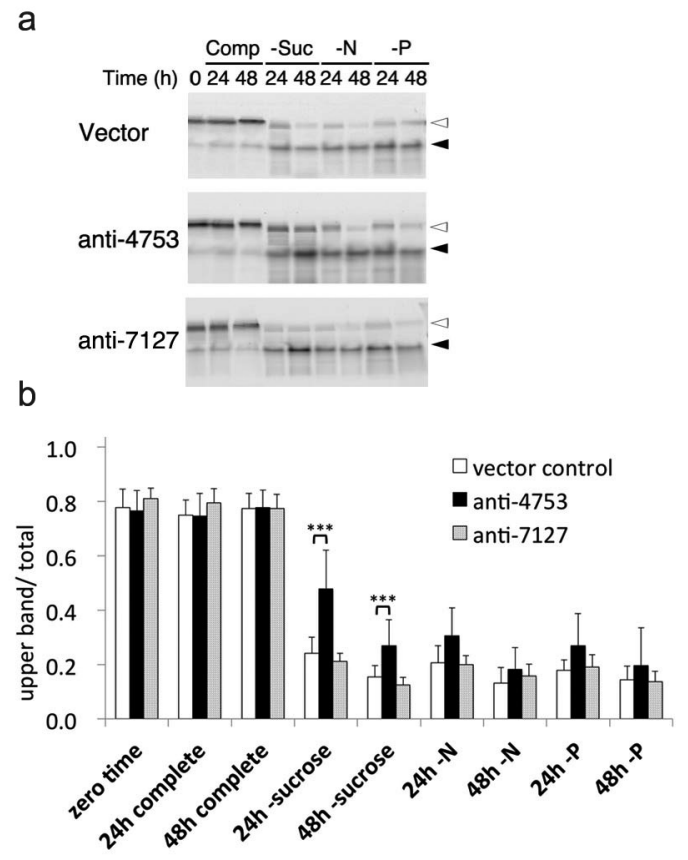

Fig. 1. Antisense suppression of the gene corresponding to EST BY4753 retards the progression of autophagy in tobacco BY-2 cells.

a. The time-course analysis of autophagy induction in BY-2 cells that were expressing both Cyt b5-RFP and the antisense construct for BY4753, BY7127 or vector control. Rapidly-growing cells in suspension were incubated in a medium either of complete formula (Comp), without sucrose $(-\mathrm{Suc})$, without nitrogen $(-\mathrm{N})$, or without phosphate $(-\mathrm{P})$ for 24 and 48 hours. Proteins in the cells were separated by SDS-PAGE and red fluorescence was recorded. Representative images are shown. Open and closed arrowheads indicate the non-processed and processed forms of Cyt b5-RFP, respectively.

b. Results of the quantifications described in Fig. 1a. Independent transformants were analyzed as described, and the ratio of the intensity of upper bands to the sum of the intensity of the upper and lower bands was quantified. The averages of twelve independent transformants are shown. Error bar, SD. Asterisks indicate a significant difference between vector control and antisense transformants using Student's $t$-test at the level of $p<0.001$. 
To confirm that the expression of the antisense construct for BY4753 retarded autophagic degradation, we cultured individual transformed colonies separately, and 12 of these were introduced into suspension and subjected to the autophagy assay as described above. We compared the effect of these antisense constructs at both $24 h$ and $48 h$ after autophagy induction. For control, we tested the antisense construct for BY7127 by the same way. Compared to vector control, introduction of the BY4753-antisense construct significantly retarded the processing of intact Cyt b5-RFP under sucrosestarved condition during $24 h$ of incubation (Fig. 1). Forty-eight hours after induction, the difference between vector control and BY4753-suppressor turned smaller but the difference was still statistically significant. In contrast, no significant difference was observed between vector control and transformants containing the BY7127-antisense construct. These observations suggest that the protein kinase that is encoded by BY4753 can modulate sugar-depletion-dependent autophagy in tobacco BY-2 cells.

\section{Analysis of the primary structure of the protein kinase encoded by BY4753}

To characterize the protein kinase encoded by BY4753, we first analyzed the entire nucleotide sequence of the EST clone BY4753 (Accession No. AB195174) and predicted the encoded protein. The encoded protein has high similarity to mammalian PITSLRE/CDK11 protein kinases. To further confirm that this cDNA encodes a homolog of mammalian PITSLRE/CDK11 protein kinases, a full-length cDNA that contained this sequence was screened from a full- length enriched cDNA library from tobacco BY-2 cells (Toyooka et al., 2009). The resulting clone of 3537 nucleotides in length (Accession No. LC136985) encodes a protein of 652 amino acids with a protein-kinase domain in the longest open reading frame. The predicted molecular mass of this protein is 73,035.

We next analyzed the similarity of this sequence to transcripts from $N$. sylvestris and $N$. tomentosiformis, the diploid mother plants of the tetraploid N. tabacum, using BLASTn. The nucleotide sequence is $>99 \%$ identical to the cDNAs of the predicted CDKG2 from $N$. tomentosiformis (NtomCDKG2) transcription variant X2 (XP_009591488.1) (Fig. 2). Thus, we named this gene $N$. tabacum cyclin dependent kinase G2 and abbreviated it as $N t C D K G 2$. The nucleotide sequence is less similar (ca. $97 \%$ identity) to its $N$. sylvestris counterpart (XP_009771046.1). This sequence difference suggests that the NtCDKG2 gene originated from $N$. tomentosiformis. This sequence is also different from the transcript variant X1 of NtomCDKG2 (XP_009591473.1) and several other variants which have identical splicing pattern with variant $\mathrm{X} 1$ in the coding region, but difference in the pattern formed in the 3' non-coding region. Analysis of the gene sequence corresponding to these transcripts in $N$. tomentosiformis indicates that these gene products are splicing variants of the same gene (Fig. 2).

Comparison of the amino acid sequence of the protein encoded by the NtCDKG2 cDNA (NtCDKG2;1) using BLASTp indicated that this protein has amino acid sequence almost identical to the predicted sequence for $N$. tomentosiformis cyclin-dependent kinase G2 isoform X2 (Fig. 3, CDKG2-isoform X2_N.tomentosiformis), and

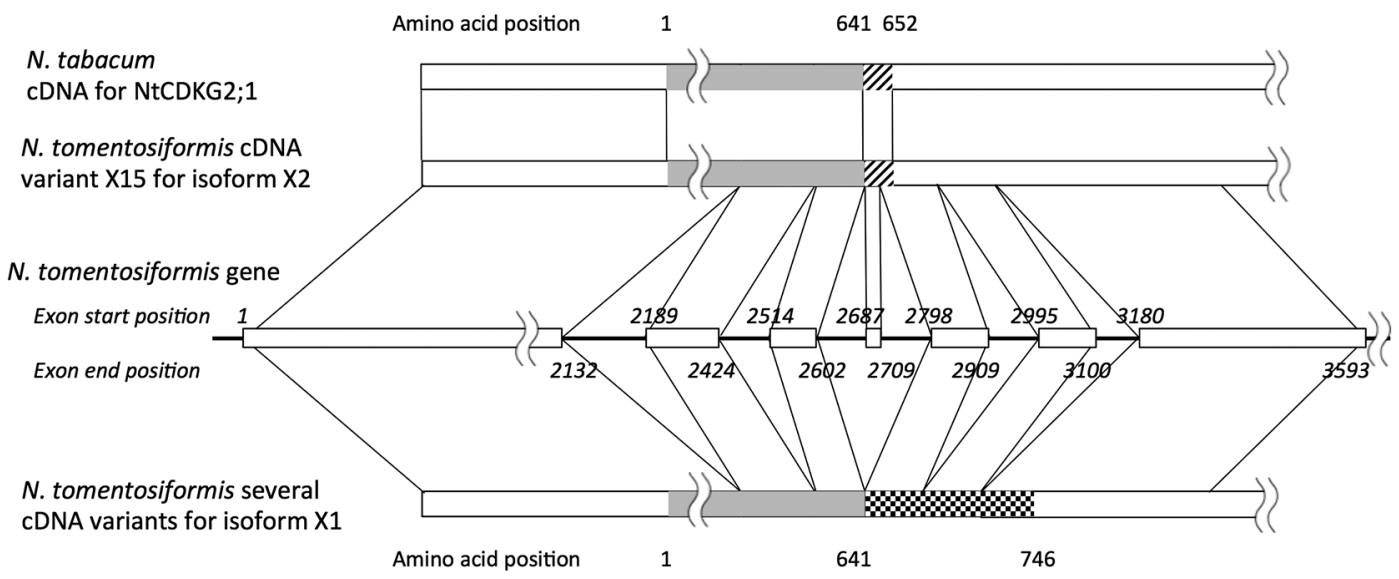

Fig. 2. Structure of the genes, mRNAs, and proteins for NtCDKG2, and related mRNAs and proteins. Schematic representation of the intron-exon comparison of cDNA for NtCDKG2 (this study) with the $N$. tomentosiformis CDKG2 mRNA variant 15 for CDKG2 protein isoform X2 (XM_009593193); part of the $N$. tomentosiformis genomic contig Ntom_contig1481 (ASAG01001481) corresponding to the 5' non-coding, coding, and part of the 3' non-coding regions of the $N$. tomentosiformis CDKG2 gene; and the N. tomentosiformis CDKG2 mRNA variants for CDKG2 protein isoform X1 (XM_009593178, XM_009593179, XM_009593180, XM_009593181, XM_009593182, XM_009593183, XM_009593184, XM_009593185, XM_009593186, XM_009593187, XM_009593188, XM_009593189, XM_009593190, XM_009593191, XM_009593192). Thick bars correspond to exons, and shadowed, hatched, and checked regions correspond to coding regions. Amino acid positions for coding regions (with the start codon as 1) are written using Roman characters, and the nucleotide positions of genes (with the nucleotide corresponding to the most 5' position of the cDNAs as 1) are written as italic. Only introns 1-7 are shown. 
CDKG2-isoform X2_N.sylvestris CDKG2-isoform X4_N.sylvestris CDKG2-isoform X2_N.tomentosi formis NtCDKG2;1

CDKG2-i soform X1_N.tomentosi formis CDKG2-i soform X1_tomato CDKG2-isoform X2_tomato CDKG2_A.thaliana CDK11B_HUMAN

CDKG2-isoform X2_N. sylvestris CDKG2-i soform X4_N. sylvestris CDKG2-i soform X2_N.tomentosiformis NtCDKG2;1

CDKG2-i soform X1_N.tomentosi formis CDKG2-isoform X1_tomato CDKG2-isoform X2_tomato CDKG2_A. thaliana CDK11B_HUMAN

CDKG2-isoform X2_N.sylvestris CDKG2-isoform X4_N.sylvestris CDKG2-isoform X2_N.tomentosiformis NtCDKG2; 1

CDKG2-i soform X1_N.tomentosi formis CDKG2-i soform X1_tomato CDKG2-isoform X2_tomato CDKG2_A.thaliana CDK11B_HUMAN

CDKG2-isoform X2_N. sylvestris CDKG2-isoform X4_N.sylvestris CDKG2-isoform X2_N.tomentosi formis NtCDKG2;1

CDKG2-isoform X1_N.tomentosiformis CDKG2-isoform X1_tomato

CDKG2-isoform X2_tomato

CDKG2_A.thaliana

CDK11B_HUMAN

CDKG2-isoform X2_N.sylvestris CDKG2-isoform X4_N. sylvestris CDKG2-i soform X2_N.tomentosi formis NtCDKG2;1

CDKG2-i soform X1_N.tomentosi formis CDKG2-i soform X1_tomato CDKG2-i soform X2_tomato

CDKG2_A. thaliana

CDK11B_HUMAN
N1

44 DFDRDRVHDRSSRDRGRLRQ-KDVKEREVINGSYRFTSSRSDSGSSDGGA 92 44 DFDRDRVHDRSSRDRGRLRQ-KDVKEREVINGSYRFTSSRSDSGSSDGGA 92 44 DFDRDQVHDRSSRDRGRLRQ-KDVKEREVINGSYRSMSSRSDSGSSDGGA 92 44 DFDRDQVHDRSSRDRGRLRQ-KDVKEREVINGSYRSMSSRSDSGSSDGGA 92 44 DFDRDQVHDRSSRDRGRLRQ-KDVKEREVINGSYRSMSSRSDSGSSDGGA 92 44 DFDRDRGHDRGGRDRGRLRQ-KDVKERDMINGSFRSMSSRSDSGSSDGDG 92 44 DFDRDRGHDRGGRDRGRLRQ-KDVKERDMINGSFRSMSSRSDSGSSDGDG 92 44 DNGKGRVSNLRHGDRDRIKSGARQEENKMVSSGFRLSKSNPGSREVFIDL 93 51 GELRDHRMEITIRNSPYRRE-DSMEDRGEEDDSLAIKPPQQMSRKEKVHH 99

N2

332 SSSRDSYQDNDLDKNDSMDVDKDRN-YDGTSVSQSDTESEDEHDSRGIPE 380 332 SSSRDSYQDNDLDKNDSMDVDKDRN-YDGTSVSQSDTESEDEHDSRGIPE 380 331 SSSRDSYQDNDLDKNDSMDVDKDRY-YDGTSVSQSDTESEDEHDSRGIPE 379 331 SSSRDSYQDNDLDKNDSMDVDKDRY-YDGTSVSQSDTESEDEHDSRGIPE 379 331 SSSRDSYQDNDLDKNDSMDVDKDRY-YDGTSVSQSDTESEDEHDSRGIPE 379 380 SCSRESYQGNELDKNGPMDVDKDRN-YDGASVSQSDTESEDEHDSRGTPE 428 380 SCSRESYQGNELDKNGPMDVDKDRN-YDGASVSQSDTESEDEHDSRGTPE 428 336 PGSRDDFEERDAVKSDKMEIDEEEHRRENSVDSLSETDSDDEYVRHETPE 385 371 VVPESRFDR---DSGESEEAEEEVGEGTPQSSALTEGDYVPDSPALSPIE 417

C1

381 PALPPQRSVNMLQGCRSVDEFERLNRIDEGTYGVVYRARDKKTGEIVALK 430 381 PALPPQRSVNMLQGCRSVDEFERLNRIDEGTYGVVYRARDKKTGEIVALK 430 380 PALPPQRSVNMLQGCRSVDEFERLNRIDEGTYGVVYRARDKKTGEIVALK 429 380 PALPPQRSVNMLQGCRSVDEFERLNRIDEGTYGVVYRARDKKTGEIVALK 429 380 PALPPQRSVNMLQGCRSVDEFERLNRIDEGTYGVVYRARDKKTGEIVALK 429 429 AALPPQRSVNMLQGCRSVDEFERLNRIDEGTYGVVYRAKDKKTGEIVALK 478 429 AALPPQRSVNMLQGCRSVDEFERLNRIDEGTYGVVYRAKDKKTGEIVALK 478 386 PASTPLRSINMLQGCRSVDEFERLNKIDEGTYGVVYRAKDKKTGEIVALK 435 418 LKQELPKYLPALQGCRSVEEFQCLNRIEEGTYGVVYRAKDKKTDEIVALK 467

631 LPGVKVN-FVKYQFNSLRKKF--PATSFTGLPVLSDAGFDLLNKLLTYDP 677 631 LPGVKVN-FVKYQ--------------LPALGDSGLIR 653 630 LPGVKVN-FVKYQ-----------_PALGDSGLIL 652 630 LPGVKVN-FVKYQ----_--_-_-_PALGDSGLIL 652 630 LPGVKVN-FVKYQFNSLRKKF--PATSFTGLPVLSDAGFDLLNKLLTYDP 676 679 LPGVKVN-FVKHQFNNLRNKFQSPTTSYMGKPDLSESGLDLLNKLLTYDP 727 679 LPGVKVN-FVKHQ----_-_-_PALGDSGLII 701 636 LPGVKVN-FVKHQYNLLRKKFP--ATSFTGAPVLSDAGFDLLNKLLTYDP 682 668 LPAVKKMTFSEHPYNNLRKRFG--------ALLSDQGFDLMNKFLTYFP 708

C2 678 EKRITADAALNHEWFREVPLPKSKEFMPTFPAQHAQDRRVRRVMKSP--- 724

Fig. 3. Comparison of the amino acid sequences of part of the predicted N. sylvestris CDKG2 isoforms X2 (XP_009771043.1) and X4 (XP_009771046.1); NtCDKG2;1 (this study: BAU68659); predicted $N$. tomentosiformis CDKG2 isoforms X1 (XP_009591473.1) and X2 (XP_009591488.1); predicted tomato CDKG2 isoforms X1 (XP_010320984.1) and X2 (XP_010320989.1); A. thaliana CDKG2 (NP_176925.1); and human CDK11B (P21127.3). Only regions containing antigen peptide sequences and junction of splicing variants were shown. Single and double underlined regions correspond to the antigen peptides for antibody used to detect the N-terminal region and C-terminal region of NtCDKG2;1, respectively. Numbers before and after amino acid sequence indicate the position of the start and end of the sequence. 
it was more distant from the corresponding protein of $N$. sylvestris, as expected by the nucleotide sequence of the transcript. Comparison of the amino acid sequence with similar proteins in several other plant species and several other organisms indicates that this protein belongs to the cyclin-dependent kinase G2 family. In all cases, these proteins have a relatively long $\mathrm{N}$-terminal region and a conserved $\mathrm{C}$-terminal region with a protein kinase domain. The N-terminal domain of NtCDKG2;1, as well as that in similar plant orthologs, is rich in Arg, Asp, and Ser residues, whereas the corresponding region of mammalian CDK11(p110) proteins is rich in Arg, Glu, and Ser residues. The N-terminal regions of these proteins are thus quite hydrophilic, with many potential protein kinase acceptor sites.

In the case of $N$. tomentosiformis, the splicing variants described above encode proteins that have different amino acid sequences at the most C-terminal portion due to splicing result in different codon frames (Fig. 3). The $N$. tomentosiformis protein from transcript variant $\mathrm{X} 1$ and several other forms commonly encode the identical amino acid sequence, and they newly named isoform X1 (Fig. 3, CDKG2-isoform X1_N.tomentosiformis). This sequence has a C-terminal stretch of 79 amino acids with clear identity to the PITSLRE/CDK11 protein kinases in mammals (Fig. 3). N. sylvestris, as well as Solanaceae crops, express NtCDKG2;1-type splicing variants with altered $\mathrm{C}$-terminal amino acid sequences (Fig. 3). In contrast, Arabidopsis thaliana expresses only a single form to the homolog to NtCDKG2 protein encoded by the At1g67580 gene. In this case, the most C-terminal portion of the amino acid sequence showed high identity to CDKG2-isoform X1 of N. tomentosiformis and mammalian PITSLRE/CDK11 kinase (Fig. 3).

\section{Characterization of NtCDKG2 protein}

The predicted amino acid sequences of NtCDKG2;1, as well as of several Solanaceae CDKG2 proteins, lack the most C-terminal conserved sequence found in mammalian PITSLRE/CDK11 protein kinases, and are predicated to be about $73 \mathrm{kDa}$ in size. In contrast, the Arabidopsis AtCDKG2 protein, as well as the CDKG2isoform X1 of $N$. tomentosiformis and proteins encoded by similar splicing variants, are predicted to include the most C-terminal sequences and to be about $84 k D a$. To determine whether the $73 \mathrm{kDa}$ form or both the $73 \mathrm{kDa}$ and $84 k D a$ forms of these proteins are expressed in tobacco BY-2 cells, we made rabbit polyclonal antibody against a mixture of two peptides corresponding to the $\mathrm{N}$-terminal region of the protein (Fig. 3, peptide N1 and N2).

The total lysate of the tobacco cells was separated into sedimented and soluble fractions by centrifugation. Thereafter, the proteins in these fractions were separated by SDS-PAGE, transferred to a PVDF membrane, and probed with antibody. Antigen-antibody complexes on the membrane were probed with fluorophore-labeled anti-rabbit IgG and recorded by fluorescence imaging.

As shown in Figure 4a, the antibody recognized polypeptides that migrated at the $73 k D a$ and $23 k D a$ posi- a

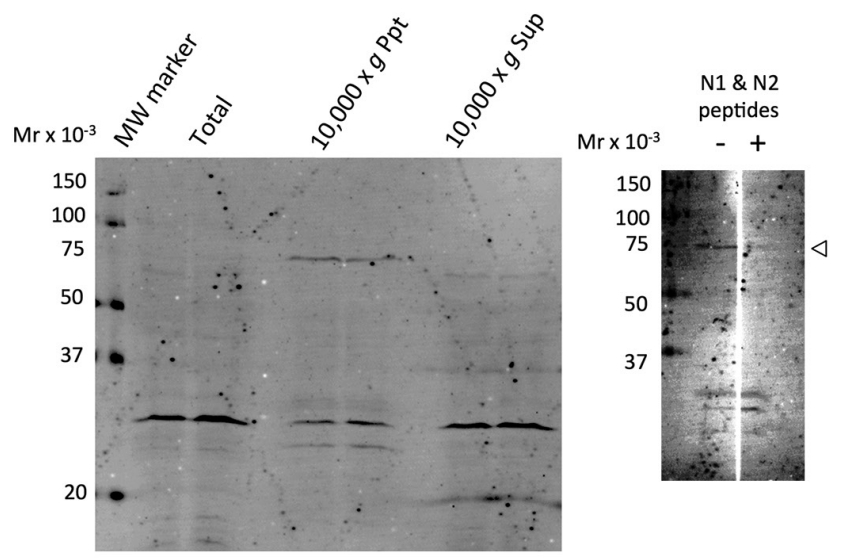

C

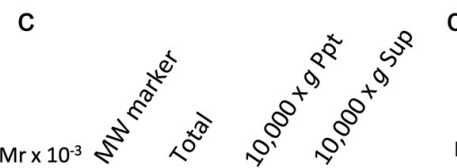

d

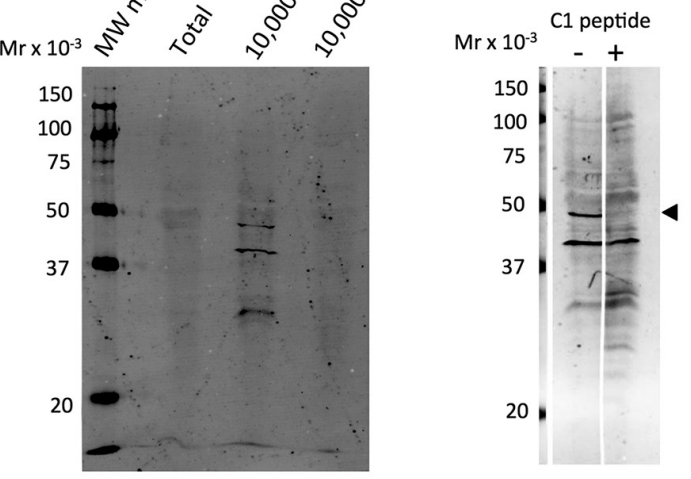

Fig. 4. Characterization of NtCDKG2;1 proteins in tobacco BY-2 cells.

a. Characterization of NtCDKG2;1 using the N-terminal antibody. Exponentially growing tobacco cells were fractionated as described in the Materials and Methods section. Equal amounts of protein $(25 \mu \mathrm{g})$ were separated by SDS-PAGE, transferred to a PVDF membrane, and probed with anti-N-terminal antibody. Duplicated samples were loaded into the gel to confirm reproducibility.

b. Confirmation of the specificity of the N-terminal antibody by antigen peptide competition. Proteins in the sedimented fraction $(10,000 \mathrm{~g}$ centrifugation of the precipitate in Fig. 3a) were separated by SDS-PAGE using a widecomb, transferred to a PVDF membrane, and the membrane was cut into two pieces at the middle of the lane. Thereafter one half was probed using the antibody as in Fig. 3a, and the other half was incubated with the same amount of antibody with an excess amount $(100 \times$ molar ratio) of antigen peptides. Then these two membranes were processed exactly the same way to detect signals. The open arrowhead indicates the $73 k D a$ band $\left(\mathrm{NtCDKG} 2 ; 1^{\mathrm{p} 73}\right)$ that was specifically weakened in the presence of antigen peptides.

c. Characterization of NtCDKG2;1 using the C-terminal antibody. Cells were fractionated as in Fig. 3a. Proteins in the fractions were separated, transferred to a PVDF membrane, and probed with anti-C-terminal antibody.

d. Confirmation of the specificity of the C-terminal antibody. Proteins in the sedimented fraction were processed as in Fig. 3b and probed with anti-C-terminal antibody, without or with an excess amount of antigen peptide. The closed arrowhead indicates the $48 k D a$ band $\left(\mathrm{NtCDKG} 2 ; 1^{\mathrm{p} 8}\right)$ that specifically disappeared in the presence of antigen peptide. 
tions in the sedimented fraction, and of $30 \mathrm{kDa}$ in all the fractions. Sometimes the antibody also recognized a polypeptide that migrated to the $32 k D a$ position, but such signals, as well as the $23 k D a$ signal, were not always observed. No polypeptides around $84 k D a$ were detected in all the experiments using this antibody. To assess whether these forms were derived from the NtCDKG2;1 protein, immunoblotting was carried out using the antibody in the presence and absence of antigen peptides. In the presence of antigen peptides, the signal of the 73 $k D a$ band extensively weakened, whereas the intensity of the other signals at low molecular weight positions were not significantly changed (Fig. 4b). These results indicate that the $73 \mathrm{kDa}$ signal should be attributed to the specific recognition by the antibody, and suggest that the NtCDKG2;1 protein expressed in tobacco BY-2 cells is the $73 k D a$ form (NtCDKG2; $1^{\mathrm{p} 73}$ ) that can be encoded by the cDNA that we isolated.

Since mammalian PITSLRE/CDK11 protein kinases have several forms (Malumbers, 2014), including the full-length form of $110 k D a$ (CDK11 $\left.11^{\mathrm{p} 110}\right)$, the smaller form $\left(\mathrm{CDK} 11^{\mathrm{p} 48}\right)$ that is generated by proteolytic processing of $\mathrm{CDK}_{11^{\mathrm{p} 110}}$ by caspase, and a $58 \mathrm{kDa}$ form $\left(\mathrm{CDK} 11^{\mathrm{p} 58}\right)$ that is generated by translation from an internal ribosomal entry site (IRES), we further investigated whether the different forms of NtCDKG2;1 protein might be present in the tobacco cells. For this purpose, we made an antibody against the $\mathrm{C}$-terminal part using a mixture of two synthetic peptides, corresponding a part of the kinase domain of the protein and a part of protein that are only present in the $84 k D a$ form (Fig. 3, peptide $\mathrm{C} 1$ and C2, respectively). This antibody also recognized proteins that were recovered in the sedimented fraction. The prominent signals in this fraction migrated to approximately the $48 k D a$ and $42 k D a$ positions, and no signal at $84 k D a$ and $73 k D a$ were observed (Fig. 4c). Sometimes this antibody also recognized a polypeptide that migrated to $28 k D a$. Immunoblotting with the antibody with the antigen peptide C1 prevented the detection of the $48 k D a$ signal almost completely (Fig. $4 d$ ) whereas $42 \mathrm{kDa}$ signal was not decreased. We thus concluded that the antibody specifically recognized a polypeptide of approximately $48 k D a$ in the sedimented fraction from tobacco BY-2 cells. A BLASTp search against both $N$. tomentosiformis and $N$. sylvestris proteins using the peptide sequence as a query detected only CDKG2 protein isoforms from these two species as complete matches. Thus, it is likely that the $48 \mathrm{kDa}$ polypeptide is a form of the NtCDKG2;1 protein (designated as NtCDKG2; $1^{\mathrm{p} 48}$ ) that was either generated from NtCDKG2; $1^{1^{73}}$ by proteolytic processing or synthesized from internal ribosome entry site in the mRNA as is the case with several mammalian PITSLRE/CDK11 forms. We had not carried out peptide competition analysis using the C2 peptide as the sequence of NtCDKG2;1 protein deduced from the sequence of corresponding cDNA does not encode a protein that contain the sequence of the $\mathrm{C} 2$ peptide.
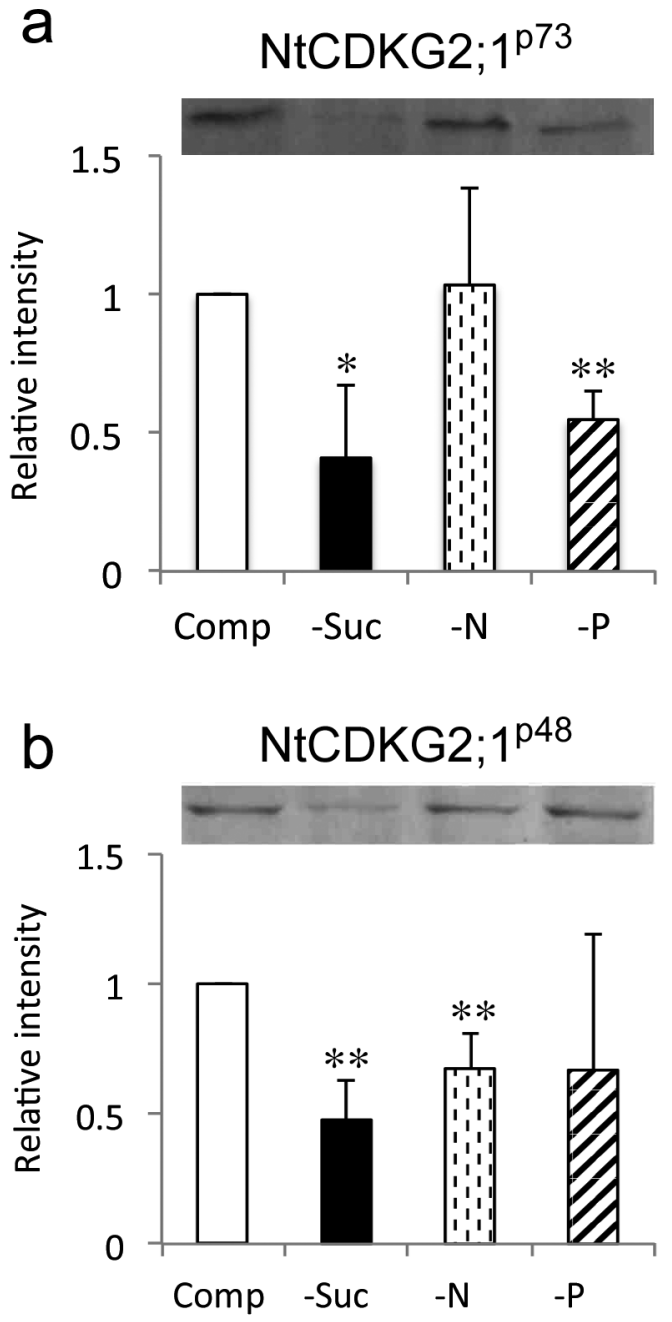

Fig. 5. Different responses of the NtCDKG2; $1^{\mathrm{p} 73}$ and NtCDKG2; $1^{\mathrm{p} 48}$ levels under nutrient deficient conditions.

a. Change in the levels of NtCDKG2;1 $1^{\text {p73 }}$ under nutrient deficient conditions. Exponentially growing cells were incubated in normal medium with either complete composition (Comp), nitrogen-free medium $(-\mathrm{N})$, sucrose-free medium (-Suc), or phosphate-free medium for $24 \mathrm{~h}$. Thereafter the sedimented fractions were prepared with the cells. Equal amount of proteins in these fractions were separated by SDS-PAGE and the amount of each form was detected as in Fig. 3a using anti-N-terminal antibody. The upper panel shows a representative result and the lower panel shows the result of the quantification of four independent experiments. Single and double asterisks indicate significant differences relative to Comp medium by Student's $t$-test ( $\left.{ }^{*}, \mathrm{p}<0.05 ; * *, \mathrm{p}<0.01\right)$.

b. Change of the levels of NtCDKG $2 ; 1^{\mathrm{p} 48}$ under nutrientdeficient conditions. Levels of NtCDKG $2 ; 1^{\mathrm{p} 48}$ in the sedimented fractions used in Fig. 4a were quantified using the C-terminal antibody. The upper panel shows a representative result and the lower panel shows the result of the quantification of four independent experiments. Single and double asterisks indicate significant differences relative to Comp medium by Student's $t$-test (**, $\mathrm{p}<0.01)$.

\section{Different nutrient starvation differentially altered the levels of two distinct NtCDKG2;1 forms}

Antibodies generated against the synthetic peptides corresponding to the $\mathrm{N}$-terminal and $\mathrm{C}$-terminal parts of NtCDKG2;1 recognized proteins of distinct sizes. To assess whether these forms have some relationship with 
the observed nutrient starvation responses, we compared the changes in the levels of these forms in cells grown in the normal medium and in the medium deficient in either sucrose, $\mathrm{N}$, or Pi. We found that the level of NtCDKG2; $1^{\mathrm{p} 73}$ decreased when cells were cultured in both sucrose-deficient and Pi-deficient media (Fig. 5a). The levels of this form in cells cultured in the N-deficient medium were indistinguishable from those observed in the complete medium. In contrast, the level of NtCDKG2; $1^{\text {p48 }}$ decreased upon depletion of either sucrose or $\mathrm{N}$, and the average level under Pi depletion was also lower than that of the control (Fig. 5b). These observations suggest that the levels of the two forms of NtCDKG2;1 protein are differentially regulated under the depletion of different major nutrients.

\section{Other cellular events responsive to nutrient deple- tion}

Since the levels of different forms of NtCDKG2;1 were not identical under different starvation conditions, we investigated whether this decrease was correlated with cellular events other than autophagy induction. We measured the mitotic index and the cell size $24 \mathrm{~h}$ after initiation of the starvation. As shown in Fig. 6a, cytokinesis was prevented in by culturing cells in medium deficient in either sucrose, $\mathrm{N}$, or Pi. In addition, cells incubated in a medium without sucrose were shorter in length (Fig. 6b).

\section{DISCUSSION}

Through screening of genes that affect the progression of autophagy using the aggregate degradation assay, we found that the expression of the antisense construct for the tobacco NtCDKG2 gene, which encodes a homolog of animal PITSLRE/CDK11 kinase, retards autophagy progression under a sucrose-starved conditions (Fig. 1). It was shown in Drosophila that CDK11 is a modulator for autophagy. In this case, depletion of CDK11 initially induces autophagy, however, at later time points, CDK11 is critically required for autophagy progression (Wilkinson et al., 2011). Since the effect of the expression of the antisense construct for $N t C D K G 2$ on retardation of autophagy was only partial (Fig. 1), and act only against sucrose depletion, CDKG2 could be a mild modulator for plant autophagy responsive to certain conditions. At present, we had not analyzed the suppression efficiency of NtCDKG2 and the efficiency of autophagy retardation, therefore, further assessments or confirmation of the putative roles for CDKG2 in modulation of autophagy in plants should be carried out in the future.

CDKG2, like its mammalian counterpart PITSLRE/ CDK11, is a member of the cyclin-dependent protein kinase family. The function of this group of protein kinases has not been elucidated, especially in plants. Recently, importance of Arabidopsis CDKG2 gene in smooth organogenic induction in vitro was implicated (Żabicki et al., 2013). Furthermore, the loss of function in the gene led to an increased tolerance to salinity
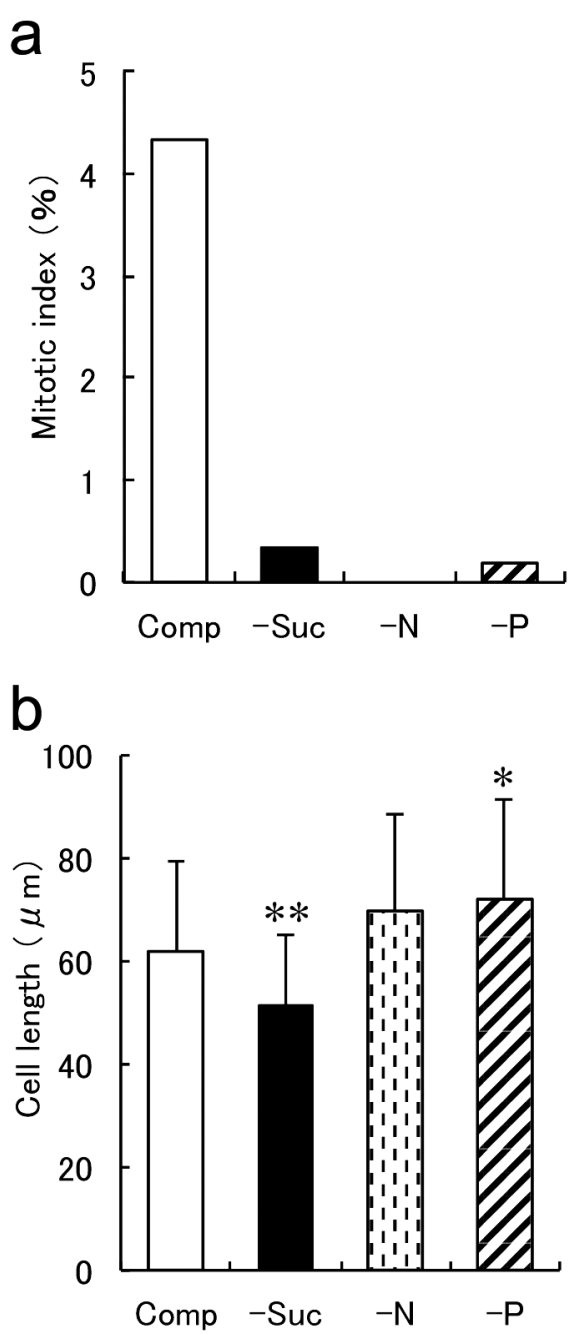

Fig. 6. Effect of nutrient starvation on cell proliferation and cell elongation.

a. Cell division is arrested under nutrient starvation conditions. Tobacco BY-2 cells in the exponential growth phase were incubated for $24 \mathrm{~h}$ in complete or nutrient-deficient medium as described in Fig. 4a. Images of the cells were then collected using a microscope and the mitotic index (percentage of cells in the mitotic phase) was measured. Experiments were repeated twice and essentially identical results were obtained. The average of the two experiments is shown.

b. The different responses of cell length under different types of nutrient starvation. Images were captured as described in Fig. 5a. The lengths of the cells in the images were measured using ImageJ software. The average of 50 cells is shown. Error bar, SD. Single and double asterisks indicate significant differences relative to Comp medium by Student's $t$-test (*, p $<0.05 ; * *, p<0.01)$.

stress and an acceleration of flowering time through upregulation of numbers of stress-responsive genes (Ma et al., 2015). This regulation might be the result of the splicing control because it has been shown recently in $A$. thaliana that CDKG2 together with its cognate cyclin, cyclin L1 is involved in thermo-sensitive alternative splicing of spliceosome component ATU2AF65A and flowering locus M transcripts (Cavallari et al., 2018; Nibau et al., 2020). It was also reported that the fusion proteins made of Arabidopsis CDKG2 and GFP were 
targeted to the nucleus (Żabicki et al., 2013). This localization pattern is consistent with our fractionation study that anti-NtCDKG2 antibodies specifically recognized proteins that were recovered in the sedimented fraction (Fig. 4).

However, the function of PITSLRE/CDK11 family proteins has not been elucidated in depth even in mammalian systems, due to the heterogeneity of the protein products and the complex roles of each form. There are three major forms of PITSLRE/CDK11 proteins in mammals: CDK11 ${ }^{\mathrm{p} 110}, \mathrm{CDK}_{1} 1^{\mathrm{p} 58}$, and $\mathrm{CDK} 11^{\mathrm{p} 48}$. The mechanisms for generation of these forms are complex because CDK $11^{\text {p48 }}$ is released after proteolytic processing, whereas $\mathrm{CDK} 11^{\mathrm{p} 58}$ is generated by the action of IRESdependent translation (Malumbers, 2014). The longest form, CDK11 ${ }^{\text {p110 }}$, has been shown to be crucial for cell proliferation and growth in human breast cancer (Zhou et al., 2015), whereas CDK $11^{\mathrm{p} 58}$ has been shown to inhibit the growth of breast cancer cells (Chi et al., 2015). In addition, CDK $11^{\mathrm{p} 48}$ has been shown to be a key mediator of cellular apoptosis via its interactions with ribosomal protein RPS8, and to inhibit the IRESdependent translation (Hao et al., 2011). Different forms of mammalian PITSLRE/CDK11 proteins thus play diversified and even opposing roles in the cells.

We show here that there are two CDKG2 protein splicing variants in Solanaceae plants (Figs. 2). Using a specific antibody against the $\mathrm{N}$-terminal region of NtCDKG2;1, we found that NtCDKG2; $1^{\text {p73 }}$, which is the exact size expected by the length of the open reading frame, was expressed in tobacco BY-2 cells. We also detected another form, NtCDKG2; $1^{\mathrm{p} 48}$, using a different antibody that was raised against C-terminal peptides. To our knowledge, this is the first study characterizing CDKG2 proteins in plants and showing that different forms exist in the same plant cells. Interestingly, the Cterminal antibody, which recognizes NtCDKG2; $1^{\mathrm{p} 48}$, did not recognize NtCDKG2;1 $1^{\mathrm{p} 73}$ (Fig. 4). One of the likely explanation is that a post-translational modification, such as phosphorylation, affects the interaction with NtCDKG2; $1^{\text {p73 }}$ and the C-terminal antibody, because the amino acid sequence of $\mathrm{C} 1$ peptide contains a serine residue that could be a target site of protein kinase as predicted by NetPhos 2.0 (http://www.cbs.dtu.dk/services/ NetPhos/). Future analysis is required in order to clarify whether phosphorylation of this site can prevent the detection of NtCDKG2; $1^{\text {p73 }}$ by the C-terminal antibody or not.

We observed that the NtCDKG2; $1^{\text {p48 }}$ level was reduced by limitation of sucrose and $\mathrm{N}$ (Fig. 5b), whereas NtCDKG2; $1^{\text {p73 }}$ level was reduced under sucrose or Pi depletion (Fig. 5a). This observation suggests that the cellular roles of NtCDKG2; $1^{\text {p48 }}$ and NtCDKG2; $1^{\text {p73 }}$ could be different. All the starvation conditions that we tested here arrested the cell cycle progression (Fig. 6a). It will thus be interesting to address in the future whether NtCDKG2; $1^{\mathrm{p} 48}$ is involved in cell cycle progression in tobacco BY-2 cells. In support of our view, the expression of the antisense construct for NtCDKG2 retarded the progression of autophagy under sucrose- starved conditions (Fig. 1). Eventually, cell elongation was inhibited under the same conditions (Fig. 6b). These observations suggest that linkage between the cellular role of NtCDKG2; $1^{\mathrm{p} 73}$ and the cell elongation during cell proliferation. It will also be interesting to test whether such a linkage represents the cellular roles of NtCDKG2; $1^{\text {p73. }}$.

\section{AUTHOR CONTRIBUTION}

K. Matsuoka formulated the research, performed computer searches and wrote the paper. K. Matsuoka and S. Asatsuma designed experiments, and M. Nagahama, M. Shimizu and S. Asatsuma carried out experiments. All authors assisted in editing of the manuscript and approved the final version.

\section{ACKNOWLEDGEMENTS}

We thank Ms. Yumiko Suzuki at RIKEN Plant Science Center for the sequence analysis of NtCDKG2 cDNA. We also thank Dr. Masaki Ito at Kanazawa University for the nomenclature of NtCDKG2. This work was supported in part by KAKENHI [JP19380045] and [JP26292194] from Japan Society for the Promotion of Science to K. M.

\section{REFERENCES}

Avila-Ospina, L., M. Moison, K. Yoshimoto and C. MasclauxDaubresse 2014 Autophagy, plant senescence and nutrient recycling. J. Exp. Bot. 65: 3799-3811

Cavallari, N., C. Nibau, A. Fuchs, D. Dadarou, A. Barta and J. H. Doonan 2018 The cyclin-dependent kinase G group defines a thermo-sensitive alternative splicing circuit modulating the expression of Arabidopsis ATU2AF65A. Plant J. 94: 1010-1022

Chi, Y., S. Huang, H. Peng, M. Liu, J. Zhao, Z. Shao and J. Wu 2015 Critical role of CDK11(p58) in human breast cancer growth and angiogenesis. BMC Cancer 15: 701

Gális, I., P. Simek, T. Narisawa, M. Sasaki, T. Horiguchi, H. Fukuda and K. Matsuoka 2006 A novel R2R3 MYB transcription factor NtMYBJS1 is a methyl jasmonate-dependent regulator of phenylpropanoid-conjugate biosynthesis in tobacco. Plant J. 46 573-592

Hao, Y., X. Kong, Y. Ruan, H. Gan, H. Chen, C. Zhang, S. Ren and J. $\mathrm{Gu} 2011$ CDK11p46 and RPS8 associate with each other and suppress translation in a synergistic manner. Biochem. Biophys. Res. Commun. 407: 169-174

Hood, E. E., G. L. Helmer, R. T. Fraley and M. D. Chilton 1986 The hypervirulence of Agrobacterium tumefaciens A281 is encoded in a region of pTiBo542 outside of T-DNA. J. Bacteriol 168: 1291-1301

Kamada, Y., T. Sekito and Y. Ohsumi 2004 Autophagy in yeast: a TOR-mediated response to nutrient starvation. Curr. Top. Microbiol. Immunol. 279: 73-84

Kato, S., K. Ohtoko, H. Ohtake and T. Kimura 2005 Vector-capping: a simple method for preparing a high-quality full-length cDNA library. DNA Res. 12: 53-62

Klionsky, D., H. Abeliovich, P. Agostinis, D. Agrawal, G. Aliev, D. Askew, M. Baba, et al. 2008 Guidelines for the use and interpretation of assays for monitoring autophagy in higher eukaryotes. Autophagy 4: 151-175

Liu, Y. and D. C. Bassham 2010 TOR is a negative regulator of autophagy in Arabidopsis thaliana. PLoS One 5: e11883

Ma, X., Z. Qiao, D. Chen, W. Yang, R. Zhou, W. Zhang and M. Wang 2015 Cyclin-dependent kinase G2 regulates salinity stress response and salt mediated flowering in Arabidopsis thaliana. Plant Mol. Biol. 88: 287-299 
Malumbres, M 2014 Cyclin-dependent kinases. Genome Biology 15: 122 .

Mathiassen, S. G., D. De Zio and F. Cecconi 2017 Autophagy and the cell cycle: A Complex Landscape. Front. Oncol. 7: 51

Matsuoka, K 2009 Chimeric fluorescent fusion proteins to monitor autophagy in plants. Methods Enzymol. 451: 541-555

Matsuoka, K., T. Higuchi, M. Maeshima and K. Nakamura 1997 A vacuolar-type $\mathrm{H}^{+}$-ATPase in a nonvacuolar organelle is required for the sorting of soluble vacuolar protein precursors in tobacco cells. Plant Cell 9: 533-546

Matsuoka, K., T. Demura, I. Galis, T. Horiguchi, M. Sasaki, G. Tashiro and H. Fukuda 2004 A comprehensive gene expression analysis toward the understanding of growth and differentiation of tobacco BY-2 cells. Plant Cell Physiol. 45: 1280-1289

Moriyasu, Y. and Y. Ohsumi 1996 Autophagy in tobacco suspension-cultured cells in response to sucrose starvation. Plant Physiol. 111: 1233-1241

Nibau, C., M. Gallemí, D. Dadarou, J. H. Doonan and N. Cavallari 2019 Thermo-sensitive alternative splicing of FLOWERING LOCUS M is modulated by cyclin-dependent Kinase G2. Front. Plant Sci. 10: 1680

Oda, Y., S. Asatsuma, H. Nakasone and K. Matsuoka 2020 Sucrose starvation induces the degradation of proteins in trans-Golgi network and secretory vesicle cluster in tobacco BY-2 cells. Biosci. Biotechnol. Biochem. 84: 1652-1666

Ohta, S., S. Mita, T. Hattori and K. Nakamura 1990 Construction and expression in tobacco of a beta-glucoronidase (GUS) reporter gene containing an intron within the coding sequence. Plant Cell Physiol. 31: 805-813

Rouached, H., A. B. Arpat and Y. Poirier 2010 Regulation of phosphate starvation responses in plants: signaling players and cross-talks. Mol. Plant 3: 288-299

Sano, T., T. Shimizu, K. Sakamoto and T. Nagata 2004 Block points in the cell cycle progression of plant cells: Deduced lessons from tobacco BY-2 cells. In "Tobacco BY-2 Cells" Biotechnology in Agriculture and Forestry 53 ed. by T. Nagata, S. Hasezawa and D. Inzé, Springer, Berlin. pp.149-158
Tasaki, M., S. Asatsuma and K. Matsuoka 2014 Monitoring protein turnover during phosphate starvation-dependent autophagic degradation using a photoconvertible fluorescent protein aggregate in tobacco BY-2 cells. Front. Plant Sci. 5: 172

Toyooka, K. and K. Matsuoka 2006 Autophagy and non-classical vacuolar targeting in tobacco BY-2 cells. In "Tobacco BY-2 Cells: From Cellular Dynamics to Omics" Biotechnology in Agriculture and Forestry 58 ed. by T. Nagata, K. Matsuoka and D. Inzé, Springer, Berlin, pp.167-180.

Toyooka, K., Y. Moriyasu, Y. Goto, M. Takeuchi, H. Fukuda and K. Matsuoka 2006 Protein aggregates are transported to vacuoles by a macroautophagic mechanism in nutrient-starved plant cells. Autophagy 2: 96-106

Toyooka, K., Y. Goto, S. Asatsuma, M. Koizumi, T. Mitsui and K. Matsuoka 2009 A mobile secretory vesicle cluster involved in mass transport from the Golgi to the plant cell exterior. Plant Cell 21: 1212-1229

Wilkinson, S., D. R. Croft, J. O’Prey, A. Meedendorp, M. O'Prey, C. Dufès and K. M. Ryan 2011 The cyclin-dependent kinase PITSLRE/CDK11 is required for successful autophagy. Autophagy 7: 1295-1301

Xiong, Y. and J. Sheen 2014 The role of target of rapamycin signaling networks in plant growth and metabolism. Plant Physiol. 164: $499-512$

Yuasa, K., K. Toyooka, H. Fukuda and K. Matsuoka 2005 Membrane-anchored prolyl hydroxylase with an export signal from the endoplasmic reticulum. Plant J. 41: 81-94

Zabicki, P., E. Kuta, M. Tuleja, K. Rataj and P. Malec 2013 Arabidopsis cyclin-dependent kinase gene CDKG;2 is involved in organogenic responses induced in vitro. Acta Biol. Crac. Ser. Bot. 55: 37-48

Zhou, Y., C. Han, D. Li, Z. Yu, F. Li, Q. An, H. Bai, et al. 2015. Cyclin-dependent kinase 11(p110) (CDK11(p110)) is crucial for human breast cancer cell proliferation and growth. Sci. Rep. 5: 10433 